Hence $\log \left(1-\frac{1}{n}\right)>-\frac{1}{n-1}$.

We have, therefore,

$$
\left|\log \prod_{p \leqq x}\left(1-\frac{1}{p}\right)+\sum_{p \leqq x} \frac{1}{p}\right|<\sum_{p \leqq x} \frac{1}{p(p-1)}<\sum_{2 \leqq n \leqq x}\left(\frac{1}{n-1}-\frac{1}{n}\right)<1 .
$$

Also $\left|\sum_{p \leqq x} \frac{1}{p}-\log \log x\right|<c_{5}$.

Hence $\left|\log \prod_{p \leqq x}\left(1-\frac{1}{p}\right)+\log \log x\right|<c_{6}$, which proves the theorem.

\title{
Trintty College,
}

DUbisn.

\section{A note on some networks of polygons}

By W. BarRetT.

Given an infinity of polygons which form the boundary of a finite number of polyhedra, we shall consider the complex $K$ consisting of the polyhedra, and of the faces, edges and vertices of the polygons. We consider only those cases in which the Eulerian Characteristic $N$ of $K$ is finite. Then if the mean number of sides meeting at a vertex is $p$, and the mean number of sides of a polygon is $q$, then

$$
\frac{1}{p}+\frac{1}{q}=\frac{1}{2}
$$

The complex $K$ is considered as the limit of a complex $K^{\prime}$ having a finite number $v_{0}$ of points, $v_{1}$ of edges, $v_{2}$ of polygons, and $v_{3}$ of polyhedra, when $v_{2}$ tends to infinity in a definite manner. Since $v_{2} \leqq \sum_{r=1}^{v_{1}}\left(\begin{array}{c}v_{1} \\ r\end{array}\right)$, which is finite if $v_{1}$ is finite, it follows that $v_{1}$ is infinite if $v_{2}$ is infinite. 
$\mathrm{x} x \mathrm{iv}$

Now by the definition of $N$,

$N=v_{0}-v_{1}+v_{2}-v_{3}$
$\therefore \frac{v_{0}}{v_{1}}-1+\frac{v_{2}}{v_{1}}=\frac{N+v_{3}}{v_{1}}$ which tends to zero as $v_{1}$ tends to infinity.

So for $K, \frac{v_{0}}{v_{1}}+\frac{v_{2}}{v_{1}}=1$.

But $v_{0} p=$ the number of lines counted twice $=2 v_{1}$, and similarly $v_{2} q=2 v_{1}$.

Therefore $\quad \frac{1}{p}+\frac{1}{q}=\frac{1}{2}$.

Corrollary. In particular the theorem applies to networks of polygons in a plane, whether the plane be considered as a numberplane, with a point at infinity $(N=1)$ or as a projective-plane with a line at infinity $(N=0)$.

Note. If the theorem is to be applied to nets of polygons on a polyhedron in cases where the network has a boundary (and in particular to plane networks of this kind) the polyhedron must be completed by the addition of a polygon whose boundary is the boundary of the net. Consider for example a circle with $n$ radii $O P_{1}, O P_{2}, \ldots, O P_{n}$ and let $n$ tend to infinity. Then to find $p$ we have $n$ lines meeting at $O$ and 3 lines meeting at each $P$, i.e., $p=(3 n+n) /(n+1) \rightarrow 4$; to find $q$ we have $n$ triangles and one $n$-gon, i.e., $q=(3 n+n) /(n+1) \rightarrow 4$, verifying the theorem.

\section{Long Drive,}

South RUISLIP. 